Abstract: The miniaturization of optical devices and their integration for creating adaptive and reconfigurable photonic integrated circuits requires effective platforms and methods to control light over very short distances. We present here several techniques and objects that we have developed to harness light at the sub-micrometer scale. These new tools include planar photonic crystal on nonlinear chalcogenide glasses, tapered silica fibres, optofluidics, and optical trapping. Their association could provide the basic building blocks of completely new architectures and platforms that would have an impact on numerous applications, from optical logic to sensing.

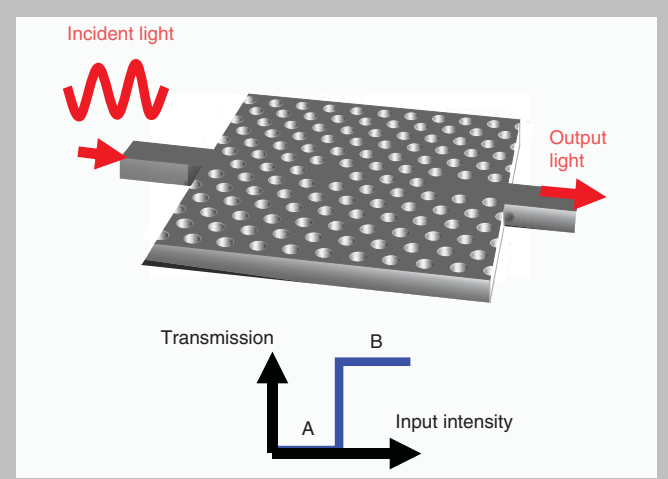

Schematic of an all-optical switch, a "photonic transistor"

\title{
Frontiers in microphotonics: tunability and all-optical control
}

C. Monat, ${ }^{1, *}$ C. Grillet, ${ }^{1}$ P. Domachuk, ${ }^{1,2}$ C. Smith, ${ }^{1}$ E. Magi, ${ }^{1}$ D.J. Moss, ${ }^{1,3}$ H.C. Nguyen, ${ }^{1}$ S. TomljenovicHanic, ${ }^{1}$ M. Cronin-Golomb, ${ }^{1,2}$ B.J. Eggleton, ${ }^{1}$ D. Freeman, ${ }^{4}$ S. Madden, ${ }^{4}$ B. Luther-Davies, ${ }^{4}$ S. Mutzenich, ${ }^{5}$ G. Rosengarten, ${ }^{5,6}$ and A. Mitchell ${ }^{5}$

${ }^{1}$ Centre for Ultrahigh-bandwidth Devices for Optical Systems (CUDOS), School of Physics, University of Sydney, Sydney, NSW 2006, Australia

${ }^{2}$ Biomedical Engineering, Tufts University, 4 Colby Str., Medford, MA 02155, USA

${ }^{3}$ Institut national de la recherche scientifique (INRS), Énergie, Matériaux et Télécommunications, Université du Québec, 1650 boulevard Lionel-Boulet, Varennes, Québec, J3X-1S2, Canada

${ }^{4}$ Centre for Ultrahigh-bandwidth Devices for Optical Systems (CUDOS), Laser Physics Center, Australian National University, Canberra, ACT 0200, Australia

${ }^{5}$ MMTC, Dept. of Electrical and Computer Engineering, RMIT University, Melbourne, VIC 3001, Australia

${ }^{6}$ School of Mechanical and Manufacturing Engineering, The University of New South Wales (UNSW), Sydney, NSW 2052, Australia

Received: 18 November 2006, Accepted: 21 November 2006

Published online: 7 December 2006

Key words: integrated optics; planar photonic crystals; chalcogenide; nonlinear optics; optofluidics; optical trapping

PACS: 42.82.-m, 42.70.Qs, 42.65.Hw, 42.81.-i, 47.85.Np, 42.65.Jx

\section{Introduction}

The evolution of photonics towards the extreme miniaturization of optical functions ultimately aims at integrating many components with various functionalities onto the same chip. To achieve this goal, novel tools must be developed and associated to manipulate and control light onto compact areas. Photonic crystals (PhCs) and more specif- ically planar photonic crystals (PPhCs), which result from the drilling of periodic air hole lattices in a thin membrane, have appeared this last decade as one promising platform to confine light paths at the sub-wavelength scale onto a planar architecture [1]. This generic building block enables the realization of various components such as waveguides, microcavities, lasers, add-drop filters, and other kinds of devices to develop an actual optical circuit integrated onto

\footnotetext{
* Corresponding author: e-mail: monat@physics.usyd.edu.au
} 
a single chip [2]. At the same time, optical tunability is necessary as more complex and dynamically active functionalities like modulators or switches are targeted. However, the degree of tunability, or the range of refractive index variation that is required to create such devices, is dramatically increased by the reduction of the corresponding device length.

Presented here are the different techniques and devices that we have developed, paving the route towards the realization of compact photonic chips with a high degree of functionality. First, the chalcogenide $\mathrm{PPhC}$ platform appears as one promising photonic architecture to confine and guide light at the sub-micron-scale, where the highly nonlinear property of the material can be exploited to achieve "active" functionalities such as low threshold all-optical switches. Second, we show how tapered silica fibres [3] offer a suitable approach to confine light at reduced scales while also providing an effective inand-out "fibre-based communication gate" between compact photonic devices and the outside world. Indeed, silica nanowires are capable of guiding light at sub-micrometer scales, while the optical evanescent tail of the guided mode, highly extended outside the nanowire, enables light transfer in or out of compact and planar photonic devices. Third, we highlight other methods to achieve tunable and reconfigurable micro-optical devices, relying on the manipulation of microfluids onto compact areas of a photonic device. The effectiveness of this emerging approach has been demonstrated through highly flexible devices, in the frame of the versatile optofluidic research field [4]. Finally, optical trapping is demonstrated as a complementary and efficient method to optically manipulate microobjects with relevant optical properties. Associated with integrated photonic devices, optical tweezers can produce a novel class of compact devices with high flexibility. We briefly discuss how these various and promising "ingredients" could be further combined to achieve entirely new and highly flexible integrated functions.

\section{Chalcogenide photonic crystal platform}

\subsection{Confining and guiding light at the sub-wavelength scale on a planar platform}

PhCs were proposed in 1987 [5,6]. The concept of photonic-band gap structures (i.e. PhCs) was introduced as a formalism to describe the localization of light and the inhibition of spontaneous radiation in these structured materials. A PhC is a class of artificially created material, in which the index of refraction varies periodically; in this respect, $\mathrm{PhCs}$ can be considered as the photonic analogues of semiconductors. When carefully designed, a complete photonic band gap appears - a frequency range where the light is precluded from propagating. This concept led to an entirely new branch of photonics that is producing a wealth of science and applications: the fabrication of $\mathrm{PhCs}, \mathrm{PhCs}$ for integrated optics [2,7], PhCs for fundamental physics (cavity quantum electrodynamics [8]), and novel phenomena (negative refraction, superprisms [9], and nonlinear optics). A promising class of $\mathrm{PhC}$ is the $\mathrm{PPhC}$. It consists of a $2 \mathrm{D} \mathrm{PhC}$ structure, such as a lattice array of air holes, which provides the mechanism for lateral confinement, etched through a slab waveguide made of a high index material for vertical confinement (Fig. 1). By introducing carefully designed defect(s) in the PPhC lattice (Fig. 1), one can create a complete new range of integrated miniaturized optical devices [2]:

- PPhC waveguides with very peculiar dispersive effects that can be exploited in the context of slow light propagation [10],

- Ultra-small and high quality factor $(Q)$ cavities [11,12] that possess the ability to trap light and can be exploited to reinforce the light-matter interaction in order to create active functionalities of optical circuitry.

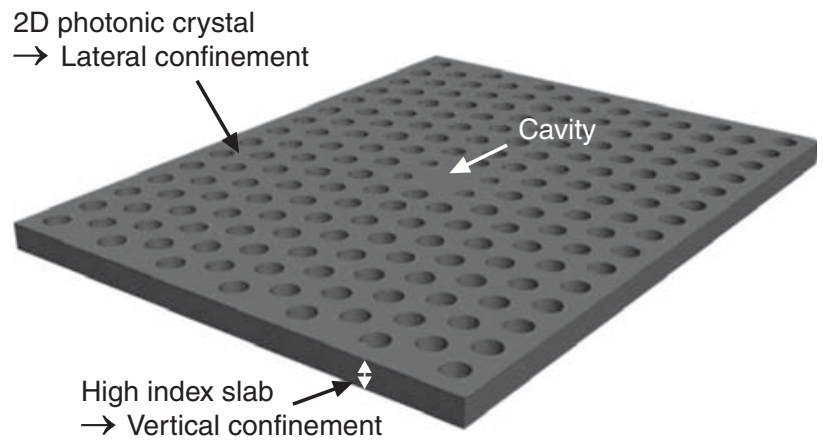

Figure $1 \mathrm{PPhC}$ cavity defect [13]

\subsection{Achieving "active" functionalities with highly non linear material: towards all-optical switching}

There have been predictions of the possibility of light switching light using nonlinear compact $\mathrm{PPhC}$ microcavities (microns in size) operating at power levels of only a few $\mathrm{mW}[14,15]$. Most experimental works that followed these predictions have been on PPhCs made from conventional optical materials [16-18]. However, little has been done on the study of highly nonlinear material in $\mathrm{PPhC}$ geometry. PPhC microcavities containing "Kerr" nonlinear material, in which the refractive index is intensitydependant, according to:

$n_{e f f}=n_{0}+n_{2} \times I$, 


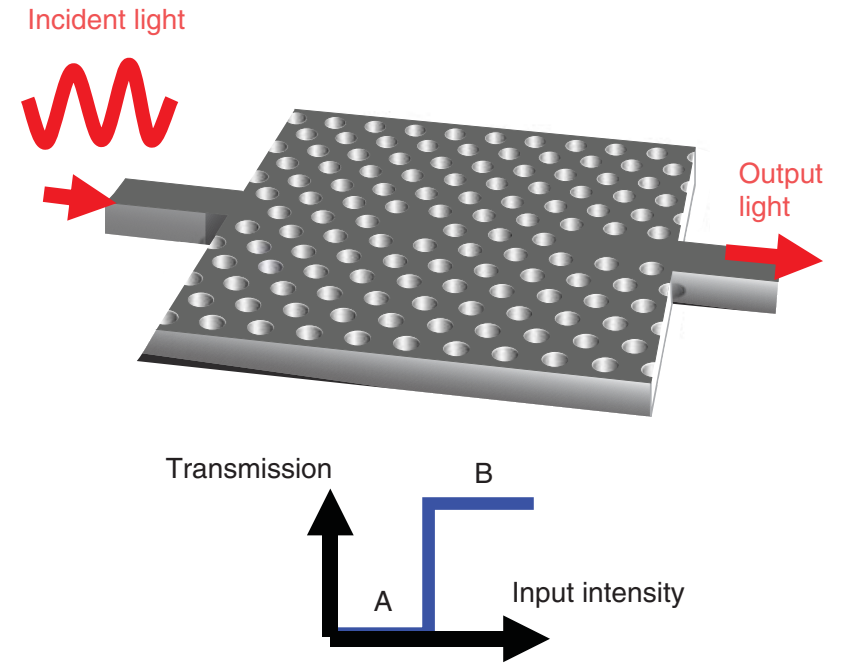

Figure 2 (online color at www.lphys.org) Schematic of an alloptical switch, a "photonic transistor". In the off-state (A), the input signal does not penetrate the gate; in the on-state (B), a control signal opens the gate through nonlinear interaction and allows the input signal to be transmitted. The control signal itself can be the input signal

where $n_{0}$ is the linear refractive index, $n_{2}$ is the nonlinear Kerr coefficient and $I$ is the electric field intensity in the cavity, are particularly promising. With increasing input power, the intensity inside the microcavity increases, magnified by the $Q$ of the resonator. This, in turns, increases the refractive index of the cavity according to (1), therefore shifts the microcavity resonance towards longer wavelengths. The transfer function (input/output characteristic) of such a system operated at a fixed wavelength can display an abrupt switching between two states (low transmission state and high transmission state) for a certain optical power threshold (Fig. 2). The threshold can be significantly lowered by using (i) a highly nonlinear material (with a high $n_{2}$ coefficient) and (ii) a high- $Q$ microcavity that enhances the light-material interaction. Such devices, whose transfer function depends on the intensity of a control signal (which can even be the information signal itself), may serve as all-optical logic gates or "photonic transistors" and be the basis of more complex all-optical logic functions.

Developing all-optical logic gates presented by the combination of PPhCs and a highly nonlinear material could open the prospect of integrated photonic devices with design flexibility. This would be highly useful for fundamentally new optical processing functionalities, including an all-optical switch array, in a considerably smaller package than that allowed by any other approach. There exists a class of material, chalcogenide glasses, which have generated great excitement recently because of their attractive properties. Chalcogenide glasses are infrared transmitting materials containing the chalcogen elements $\mathrm{S}$, Se, or
Te, compounded with network forming elements such as $\mathrm{As}, \mathrm{Si}$, and $\mathrm{Ge}$. Glasses can be formed over a wide range of compositions. The refractive index of chalcogenide is high, typically between 2.4 and 3.0, allowing PPhCs to be created. Absorption losses are low over a wide wavelength range (near- to mid-infrared). Chalcogenide glasses possess a relatively large third-order optical nonlinearity $(100-1000$ times that of silica), and low two-photon absorption. Figures of merit $\left(\mathrm{FOM}=n_{2} \beta \lambda\right.$, in which $\beta$ is the two-photon absorption coefficient) higher than 12 have been achieved in $\mathrm{As}_{2} \mathrm{~S}_{3}$ at the telecommunication wavelength. In addition to reducing the switching power requirements, the pure Kerr-like nonlinearities offer the potential for near instantaneous response times $(<100 \mathrm{fs})$ that could be the basis of fast optical switches only limited by the resonator $Q$-factor. This compares favourably to carrier-based nonlinear effects, as exploited in silicon $[16,17]$ or III-V-based devices [18], which rely on dissipation to occur after switching, limiting the response time to tens of picoseconds. In addition to that, chalcogenide glasses can be processed using conventional lithographic techniques. These properties of chalcogenide glasses along with the $\mathrm{PPhC}$ ability to confine light at the wavelength scale and to enhance light nonlinear material interaction suggest that these two ingredients are expected to become essential for all-optical ultra-fast switching at low powers.

In Sec. 3.2 we report on characteristics of $\mathrm{PPhC}$ waveguides and resonators fabricated in free standing chalcogenide glass membranes by focused ion beam (FIB) milling [19,20].

\section{Silica tapered nanowires}

\subsection{Confining and guiding light at the sub-micron scale in fibre-based systems}

Another new and powerful approach to conducting light at micron scales has recently emerged. Ultra small silica waveguides, or optical nanowires relying on taper drawing of conventional optical fibres [21], are able to guide light at visible and infrared with low loss. The tapering technique, which in our case is known as a "flame brushing" technique, consists in eating a conventional optical fibre using a butane flame while the fibre is drawn apart at either side. The taper length and profile is tailored by the appropriate choice of flame brushing profile and rate of elongation.

This technique can give rise to fibres tapered down to sub-wavelength dimensions. In this case the overall reduced taper acts as the core with the surrounding air becoming the cladding. The air cladding mode of the tapered fibre now exhibits an evanescent tail, which expands out of the silica waveguides and far into the surrounding air.

At the same time, because light transport is ensured by evanescent wave guiding, the ratio of light in the evanescent field becomes high enough to respond to the surrounding environment, making tapered nanowires partic- 


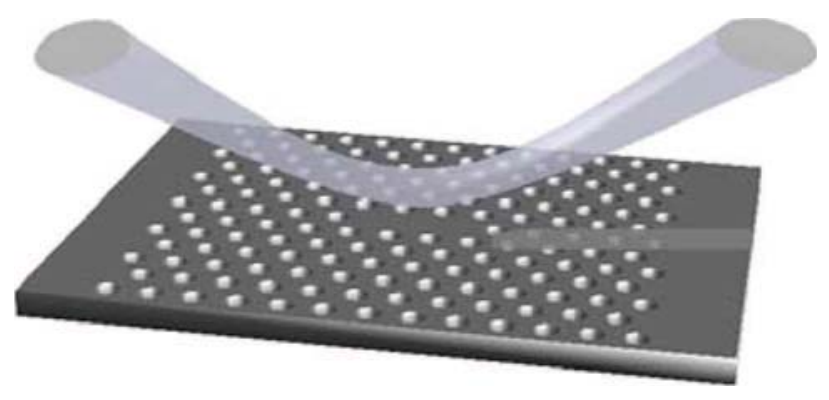

Figure 3 (online color at www.lphys.org) Schematic of the scheme used to couple light between a tapered fibre and a PPC nanocavity

ularly promising for developing miniaturized optical sensors with high sensitivity [22], but also as stated in the introduction can provide a very effective in-and-out "fibrebased communication gate" between compact photonic devices and the outside world.

\subsection{Communicating between compact photonic devices and the outside world through tapered fibres}

Communicating with waveguides and cavities with very small mode field dimensions, for instance $\mathrm{PPhC}$ waveguides and cavities, has long proven to be an insurmountable challenge. One effective approach recently developed to coupling to ultra-small devices is evanescent coupling via silica nanowires [12,23-25]. For conditions where the propagation constant of the mode in the taper and $\mathrm{PPhC}$ are matched, power can couple from the fibre to the $\mathrm{PPhC}$ structure, exciting modes of the PPhC. This is observed as a dip in the transmission spectrum through the fibre taper. Testing was performed by tapering a section of conventional single mode optical fibre to a diameter of $\approx 1 \mu \mathrm{m}$ and then forming the taper into a curve or loop shape that is $400-500 \mu \mathrm{m}$ in diameter to restrict coupling to the region of the waveguide or nanocavity (Fig. 3).

Various Chalcogenide PPhC structures have been realized and optically probed with the evanescent coupling technique, demonstrating the versatility of the evanescent coupling method. Two such PPhC structures investigated were i) long truncated waveguides formed by leaving out a row of holes from the lattice, where the structures were terminated before the edge of the lattice to create end mirrors, and ii) nanocavities formed by removing three adjacent holes from the lattice and shifting the positions of the remaining holes at each end of the nanocavity to increase the resonator $Q$-factor.

In the PPhC waveguide case (Fig. 4a), effective coupling could be obtained when the fibre was in contact with the $\mathrm{PPhC}$, leading to a maximum depth in the transmission (a)

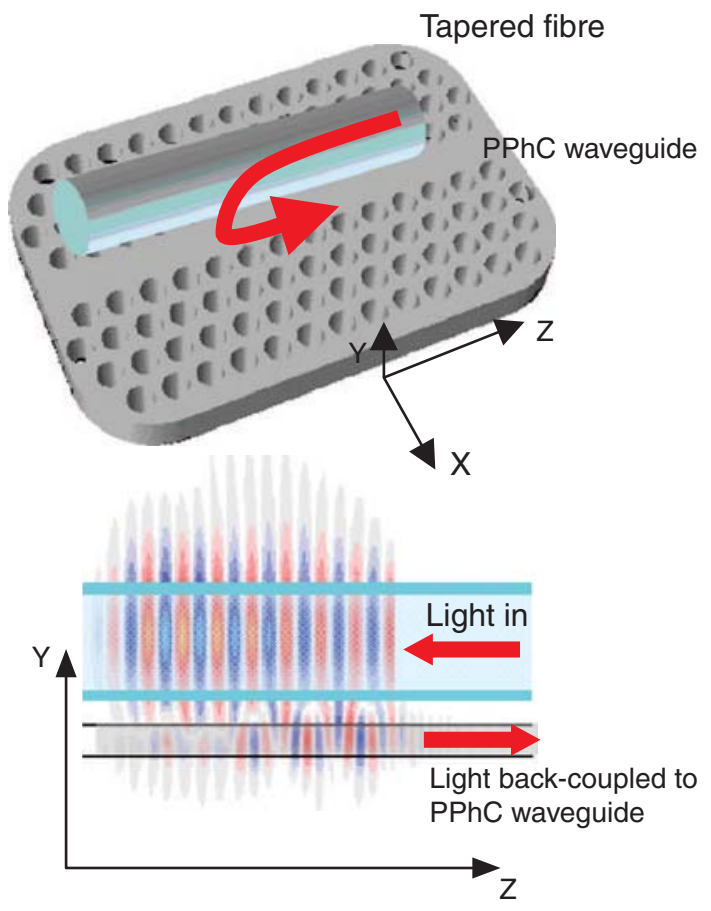

(b)

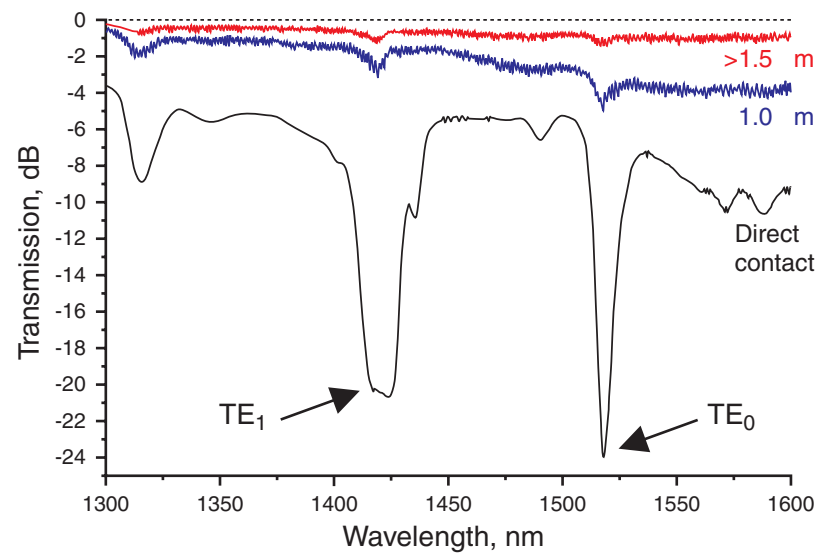

Figure 4 (online color at www.lphys.org) (a) - schematic showing the coupling from a tapered fibre to a PPC waveguide; (b) - transmission spectra through the waveguide/tapered fibre coupled system as a function of fibre to PPhC separation on the input side of the $\mathrm{PPhC}$ waveguide

dip associated with the coupling resonance of $-18 \mathrm{~dB}$, corresponding to $\approx 98 \%$ coupling efficiency (Fig. $4 \mathrm{~b}$ ). Fig. $4 \mathrm{~b}$ also displays the variation in coupling strength with fibre to $\mathrm{PPhC}$ separation.

Coupling to nanocavities was achieved by using the looped nanowires. The measurements were taken with the nanowire in direct contact and parallel to the cavity axis. Resonances were observed in the transmission spectrum around $1640 \mathrm{~nm}$. We observed the expected change in po- 


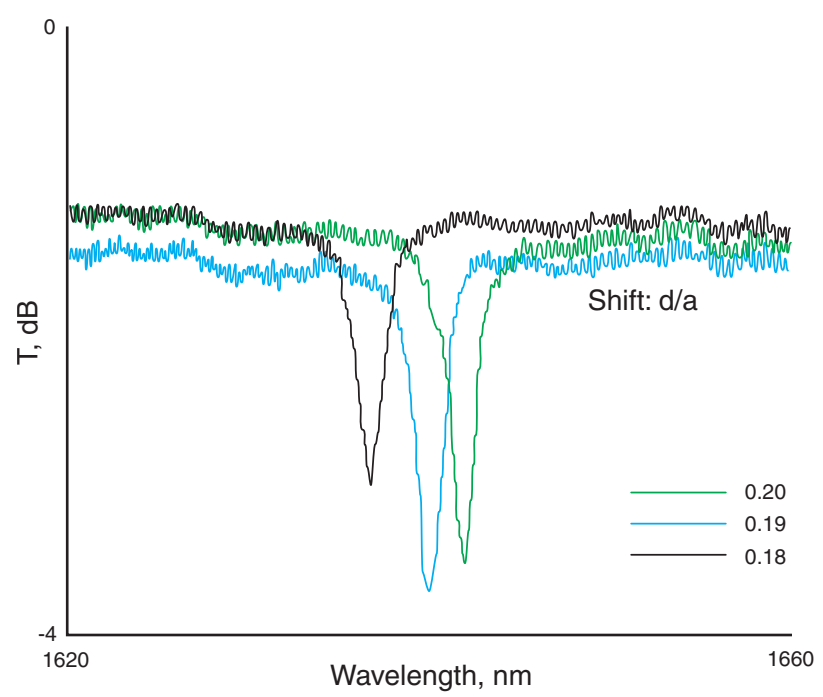

Figure 5 (online color at www.lphys.org) Transmission spectra for coupling to three different $\mathrm{PPhC}$ nanocavities, each trace corresponding to a varying displacement $d$ of adjacent holes to the cavity

sition of the resonances corresponding to the shift $d$ of the end holes, which alters the effective length of the cavity [26] (Fig. 5). The resonances displayed measured $Q$ values ranging from $500(d / a=0.18 a)$ to $800(d / a=$ $0.2 a$ ) and a corresponding transmission dip ranging from $-1.7 \mathrm{~dB}$ to $-2.2 \mathrm{~dB}$.

We have demonstrated that by using evanescent coupling from a tapered optical fibre, we can excite propagating waveguide modes as well as the high- $Q$ nanocavity modes within PPhC made from highly nonlinear chalcogenide glass. This scheme provides a promising platform to realize low power integrated all-optical switching and logic functions.

\section{Optofluidics}

Optofluidics, or the combination of photonics and microfluidics onto the same platform, appears as an interesting alternative or complementary approach to the above techniques for realizing complex and compact optical functionalities. As photonic devices are shrunk in size, the index range that is necessary to achieve the modulation of the optical functions becomes larger (unless high $Q$ microcavities are exploited as discussed in the Sec. 2). The harnessing of microfluids within a photonic chip could provide the desirable optical tunability even on a very compact space.

Advanced techniques and devices, such as micropumps, microvalves, microchannels, to confine and manipulate fluids at the microscale onto a planar and integrated architecture have been largely developed this
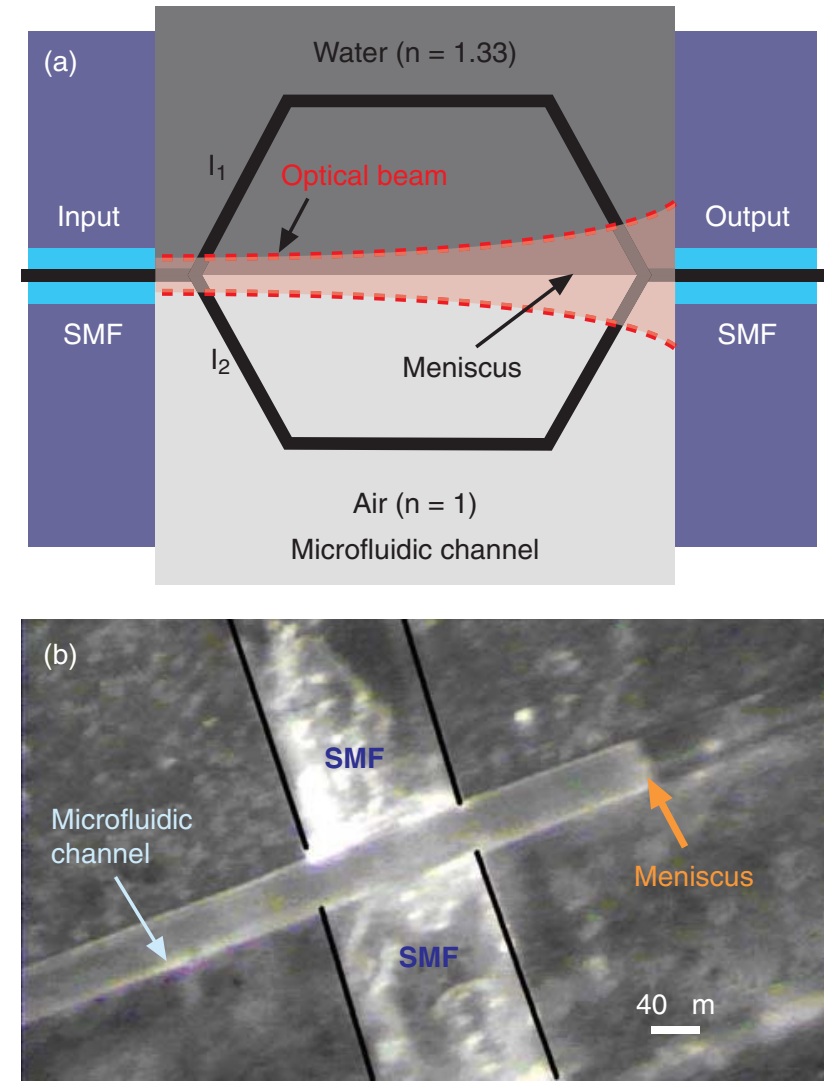

Figure 6 (online color at www.lphys.org) (a) - a top schematic representation of the single beam fluid Mach-Zehnder interferometer. The beam path through the channel is highlighted in red and superimposed on a schematic representation of a traditional Mach Zehnder interferometer, where $I_{1}$ and $I_{2}$ are the two split and delayed beams; (b) - top view photograph from the final device, consisting in two self aligned buried SMFs separated by a microfluidic channel that contains a mobile air/water meniscus

last decade [27]. These specific developments have been mainly driven by biotechnology through the realization of miniaturized lab-on-a-chips, for small volume and fast bioanalysis applications. The idea of optofluidics is to exploit these designs and fabrication techniques to build sophisticated fluid control platforms to be combined with photonic integrated architectures.

Three main properties of microfluids can be advantageously used to provide photonic devices with tunability, reconfigurability and flexibility: high refractive index modulations $(\Delta n \sim O(0.1)$ ) through fluid concentration variations, fluid mobility and flexible liquid/liquid or liquid/air interfaces. The characteristics of fluids confined at the micron-scale, such as laminar flows or the importance of surface phenomena (for instance, capillary driven forces) can also be exploited to these aims. Novel optofluidic functionalities with a high degree of flexibility have been recently demonstrated, such as tunable optical atten- 


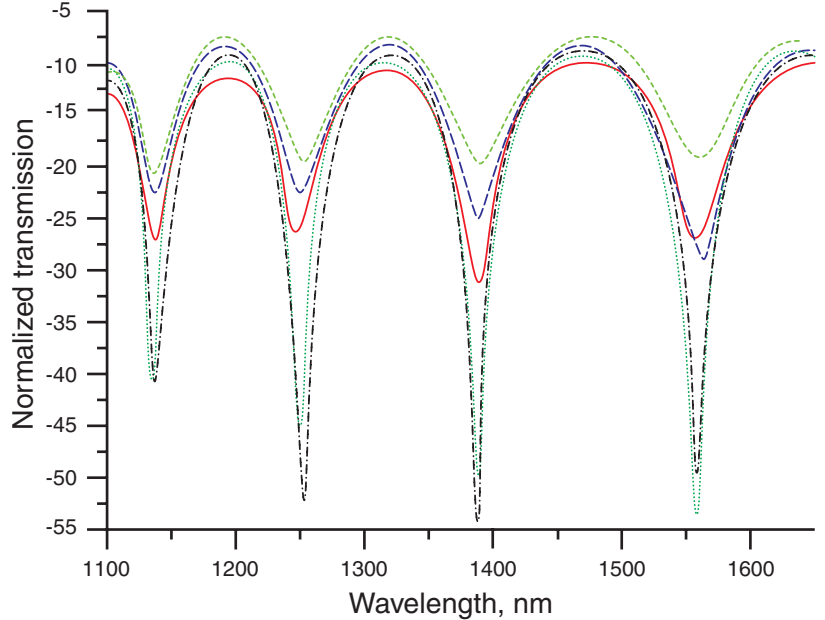

Figure 7 (online color at www.lphys.org) Experimental transmission of the device obtained for different locations of the meniscus across the beam, in front of the two single mode fibres

uators [28], reconfigurable optical filters [29-31], optical switches [32,33], liquid core/liquid cladding waveguides [34,35], or tunable microfluidic light sources and lasers $[36,37]$. Although the integration of microfluidic and photonic devices is still in its infancy, several demonstrations have recently achieved a complete integration of both sophisticated architectures for manipulating microfluids and light $[31,32,38,39]$.

To illustrate the effectiveness of this approach, we present here two optofluidic realizations. The first device relies on the interaction of an air/water meniscus with an optical channel to demonstrate tunable optical filtering onto highly compact lengths. The second device exploits photonic crystal structures and fluid interactions to produce optical switching functionality.

\subsection{Microfluidic integrated single beam interferometers}

The single beam interferometer produces a spectrally modulated response as a result of the phase delay induced between the two sections of the same beam. Indeed, when the one beam interacts with an interface made from two distinct media with different refractive index, the two sections that propagate through the respective media undergo some relative delay [40] (Fig. 6a). Our microfluidic version of the device exploits an air/water meniscus as the interface. This enables the integration of the device by combining the optical channel with a microfluidic channel onto the same platform. The high index difference displayed at the interface gives rise to a modulated response for high compact lengths $(40 \mu \mathrm{m})$. Furthermore, the actuation of the mobile meniscus provides a suitable means to tune the device response.
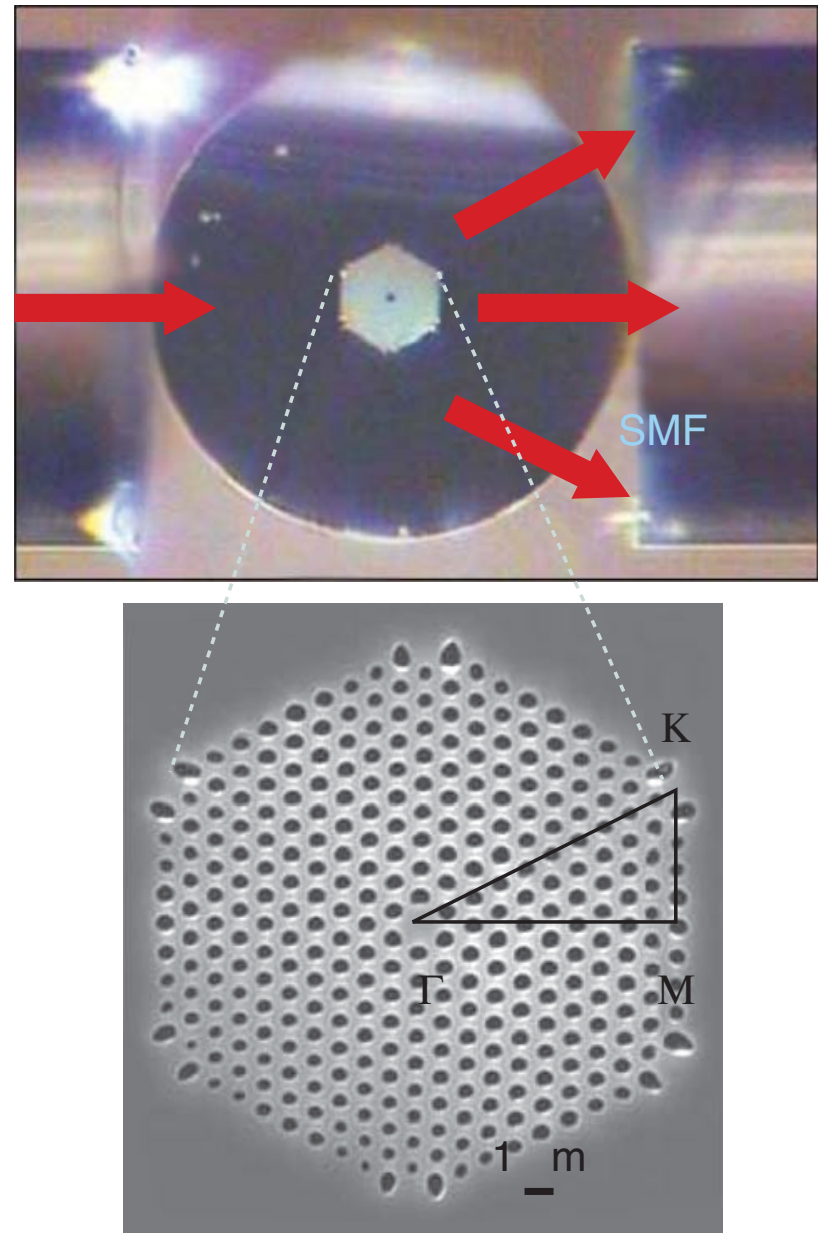

Figure 8 (online color at www.lphys.org) Photograph of the transversely probed PCF. Light incident on the side of the PCF interacts with the $\mathrm{PhC}$ region and is diffracted to various orders on the right hand side. Inset shows a scanning electron microscope image of the end-face of the PCF

Practically, the device has been fabricated by integrating a sectioned single mode fibre (SMF), embedded in UVcured polymer, with a $40 \mu \mathrm{m}$ wide perpendicular microfluidic channel onto a glass substrate (Fig. 6b). De-ionized water is introduced into the channel that is sealed by a top silicone layer. The air/water meniscus can be moved using a syringe pump in front of the two self-aligned SMFs on either side of the microfluidic channel. A broadband light source is launched into the input SMF of the device. The direct interaction of the light and the meniscus produces a spectrally modulated response with a high extinction ratio of $40 \mathrm{~dB}$ (Fig. 7). As the meniscus is displaced across the optical beam, the contrast of the device response varies strongly [40]. This modulation is achieved for a restricted displacement of the meniscus across the beam $(2-3 \mu \mathrm{m})$ and very small corresponding amounts of displaced fluid (a few $\mathrm{pL}$ ). 

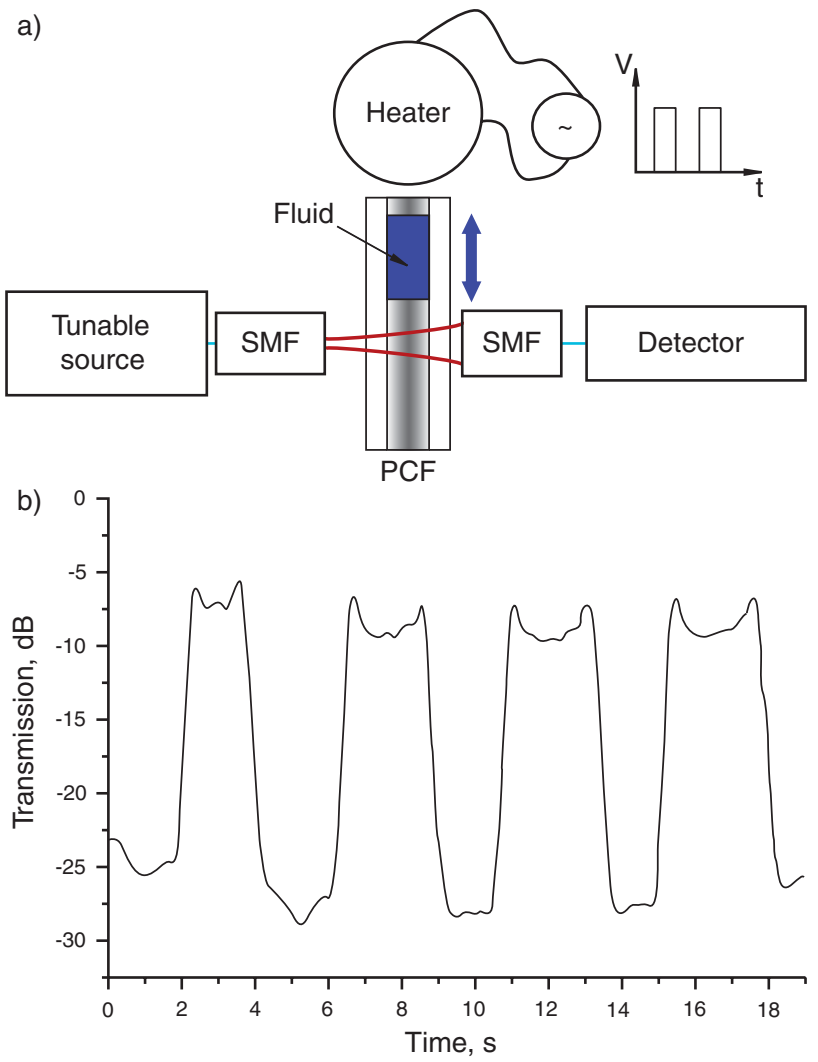

Figure 9 (online color at www.lphys.org) (a) - schematic of the setup including a heater driven by a step voltage of $4.5 \mathrm{~s}$ time period to thermally actuate a silica matching oil plug back and forth along a transversely probed PCF; (b) - corresponding response of the device, obtained at $1.5 \mu \mathrm{m}$, showing a periodic switching of the transmission. The two states correspond to the transparent state of the PhC device (on-state) when the fluid plug is moved inside the optically probed area and the transmission of the empty $\mathrm{PhC}$ fibre (off-state)

The device response proves to be very sensitive to both the meniscus location and shape [40]. Specific surface treatments (like silanization [41] or Teflon coating [42]) ensure the flattening of the meniscus, which is crucial to the device performance. Combined with microfluidic valves and electro-wetting techniques [43] to dynamically tune the meniscus geometrical properties, this new class of device could be used as an accurately tunable and compact integrated optical filter. Since the response also depends on the fluid index, the wavelength of the resonance could be tuned using an electro-optic liquid, such as liquid crystals [44].

\subsection{Optical switching with fluid infiltrated transverse photonic crystal fibres}

As a second optofluidic example, optical switching has been demonstrated, based on the displacement of a mobile fluid plug inserted within a photonic crystal fibre (PCF). PCFs contain air inclusions along their longitudinal direction (Fig. 8). When probed in their transverse direction, they display optical properties like photonic bandgaps that are similar to those displayed by the PPhCs highlighted in the first section [45]. As PCFs are commercially available and can be filled with various fluids using capillary action, they provide a convenient platform to study the interaction of fluids and light through photonic crystal structures.

As a first step, the spectral tuning of the transverse PCF photonic band-gap is demonstrated experimentally by subsequently filling the PCF with different fluids [46]. Indeed, the PCF properties depend on the refractive index contrast between the inclusions (here infused with various fluids) and the surrounding matrix of the PCF.

As a second step, using the reconfigurable properties of those fluid infused transverse PCFs, dynamic optical modulation is demonstrated [33]. The PCF is filled with a plug of silica index matching oil. Because of its refractive index (1.5) closely matched to the silica index, the oil renders the PCF roughly transparent and makes the photonic band-gap disappear. The plug is thermally actuated back and forth along the PCF, and across the transversely probed area of the PCF, using a heater driven by a periodic squarelike voltage (Fig. 9a). The transverse optical response of the device is periodically switched with a strong extinction ratio of $20 \mathrm{~dB}$ between $\mathrm{ON}$ and OFF states (Fig. 9b). The timescale of the response limited to several seconds was mainly due to the thermal actuation of the device. Other methods, like electrokinetics and electrowetting, exploiting electric field manipulating charge transport could move the microfluid at milliseconds timescales [47].

This first implementation of microfluids and photonic crystals shows the high potential of this combination to achieve novel functionalities. An integrated platform of this concept has been recently demonstrated, by combining a silicon $\mathrm{PPhC}$ waveguide and a silicone microfluidic chip, bonded together [32].

By integrating a microfluidic chip for controlling and manipulating fluids with a $\mathrm{PPhC}$ platform, as in Sec. 2, interesting functionalities could potentially be achieved. The ability to fill selectively specific row of holes with fluids opens the way to the realization of fully reconfigurable optical paths as well as reversible light traps within a photonic circuit.

\section{Optical trapping to manipulate on-chip light beams}

Optical trapping appears as another highly useful tool for the control of light at the sub-wavelength scale. Optical trapping refers to the optical manipulation of nano- and micro-scale objects through intense and tightly focused laser beams. It was demonstrated in 1986 that strongly focused laser beams provide sufficient gradient forces to 

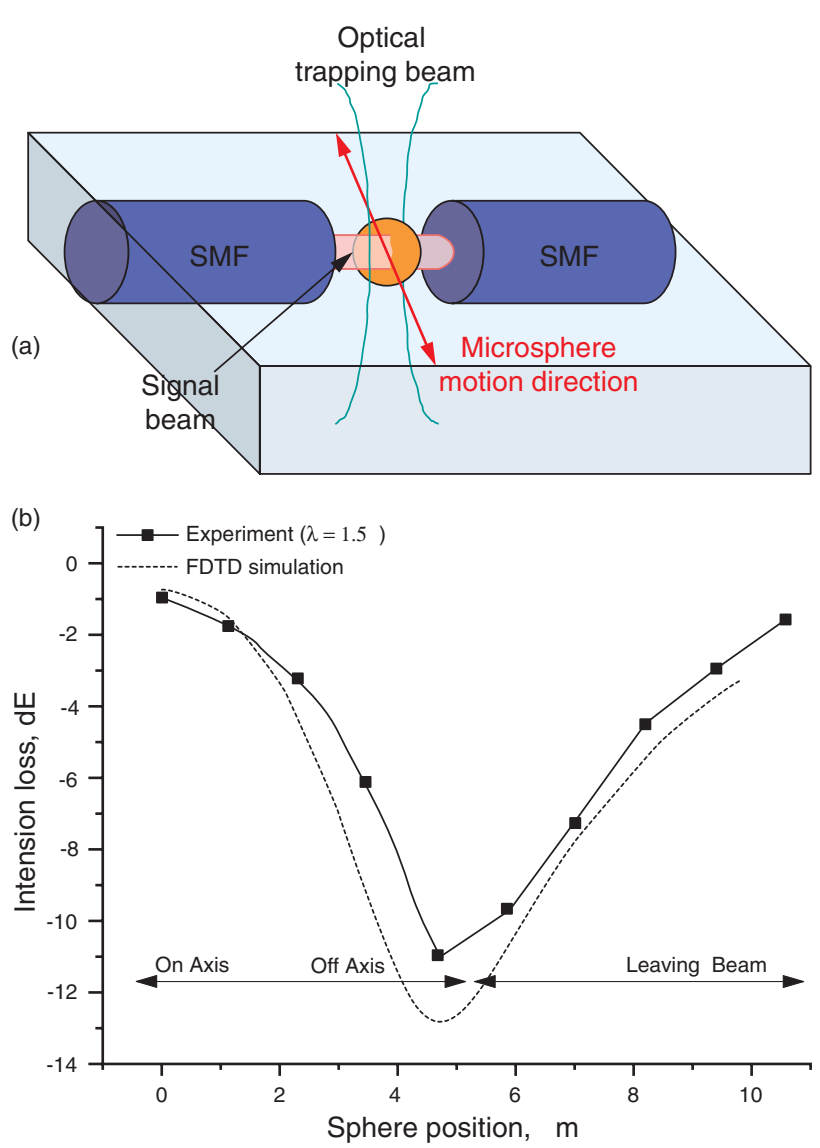

Figure 10 (online color at www.lphys.org) (a) - schematic of the device; (b) - measurements (solid line) along with FDTD simulations of the signal beam transmission at $\lambda=1.5 \mu \mathrm{m}$ as the sphere is displaced perpendicularly to the signal beam direction from on-axis (position $=0 \mu \mathrm{m}$ ) to off-axis positions

efficiently trap a nano- or micro-object around the laser focus point [48]. The creation of actual three-dimensional optical traps, even in aqueous environment, provides full control of the trapped particle location.

Whereas optical trapping has been extensively used for biological studies as a non invasive technique to manipulate cells [49], it can also be applied to the displacement of, for instance, silica microspheres within planar photonic platforms. When embedded in water, silica microspheres provide a refractive index contrast that is sufficient to make them act as microlenses at telecom wavelengths. These optically controlled lenses can be effectively used to directly manipulate light on a chip. We have demonstrated novel devices, namely tunable attenuators, by exploiting this effect [50].

The first realized device consists of two fibres on either side of a $80 \mu \mathrm{m}$ wide microfluidic channel, in which a solution with $13 \mu \mathrm{m}$ wide diluted silica microspheres is introduced (Fig. 10a). A $40 \times$ microscope objective with 0.65 numerical aperture is used in an inverted microscope setup

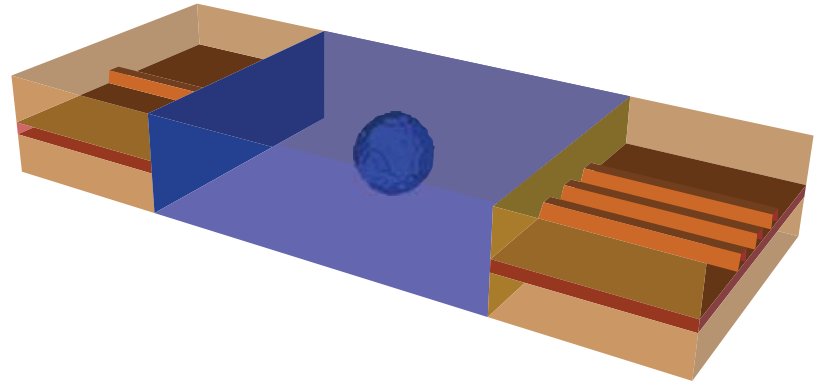

Figure 11 (online color at www.lphys.org) Schematic of the $1 \times$ 3 coupler, displaying one input rib waveguide and three output rib waveguides, on either side of a microfluidic channel containing a microsphere

to focus an intense laser beam generated by a mode locked, $\mathrm{Nd}: \mathrm{YVO}_{4}$ laser at wavelength $1064 \mathrm{~nm}$ with $\mathrm{TEM}_{00}$ profile and $0.8 \mathrm{~W}$ average power in the microfluidic channel. This relatively low numerical aperture objective and single beam configuration enables 3D trapping of a silica microsphere by using the sphere weight to compensate for the scattering force that usually tends to push the object out of the trap along the direction of the beam. By tuning the focus point along the vertical direction, the trapped microsphere can be lifted up to the level of the optical channel so that the lens interacts with the on-chip beam coming out of the fibre. By displacing laterally the microsphere across the beam path, the device transmission is attenuated (Fig. 10b). Indeed, when displaced off-axis respectively to the fibres, the silica lens deviates the beam path, reducing the amount of light that is eventually collected by the output fibre. Beyond $5 \mu \mathrm{m}$ displacement, a further increase of the microsphere lateral displacement reduces the interaction of the beam with the lens, increasing the amount of light directly transmitted through the output fibre.

The manipulation and optical control of light beam onchip could be further exploited to produce more complex functionalities like optical routers. The optically controlled lens could drive the on-chip beam to be collected through one of multiple available output channels. Although the fibre platform is not compact enough to exploit the small beam deviations produced by the trapped microlens, a waveguide platform, containing close output optical channels, could realize the scope.

Fig. 11 shows an illustration of a $1 \times 3$ coupler, which will represent a first proof of concept: the device includes an input waveguide and three output waveguides on either side of a $200 \mu \mathrm{m}$ wide microfluidic channel.

\section{Future outlook}

The ultimate vision of an adaptive and reconfigurable photonic integrated circuit is yet to occur. Advances in the development of novel components for optical interconnec- 
tion, storage, coding at the chip level etc are still required. The main difficulty will be our ability to cascade several optical functions and to combine several ingredients to make photonic integration a reality. The different techniques and devices that have been separately developed have been partly combined thus far. Nanowires have been used to probe the optical properties of chalcogenide $\mathrm{PPhC}$ waveguides or cavities. Optical trapping has been associated with an optofluidic platform to demonstrate tunable attenuators. It is reasonable to consider that these tools could be further and more deeply associated. For instance, the confined optical field of the PPhC cavities could be directly exploited to trap nano-objects in their surrounding [51], providing optical actuation and control directly onchip [52]. The immersion of the PPhC device or its integration with a microfluidic circuit could provide a convenient platform to supply the nano-objects embedded in fluid at the targeted location. The nanowire could be used to excite different optical modes of the PPhC device, inducing in turn different spatial arrangements of the nano-objects onto the device surface. In addition, the combination of microfluidics with compact photonic platform or tapered fibres offers novel opportunities for the creation of both ultra-small and sensitive devices in the context of sensing applications. In particular, the use of optical resonant microstructures, such as microcavities, can enhance locally the interaction between a solution to analyze and the optical field, in such a way that sensors with high detection limits can be constructed $[53,54]$. As novel tools are developed and become mature, new ways and opportunities to combine them appear. Such level of integration/ association should lead to significant advances in the realization of fully reconfigurable photonic circuits and highly compact sensors or lab on a chip devices with unprecedented performance.

Acknowledgements This work was produced with the assistance of the Australian Research Council under the ARC Centres of Excellence program. CUDOS (the Centre for Ultrahigh bandwidth Devices for Optical Systems) is an ARC Centre of Excellence. This work was also partly funded under ARC research grant DP0556781 (Microfluidic photonics).

\section{References}

[1] T.F. Krauss, R.M. De La Rue, and S. Brand, Nature 383, 699 (1996).

[2] M. Notomi, A. Shinya, S. Mitsugi, E. Kuramochi, and H.Y. Ryu, Opt. Express 12, 1551 (2004).

[3] P. Domachuk and B.J. Eggleton, Nature Mater. 3, 85 (2004).

[4] D. Psaltis, S.R. Quake, and C. Yang, Nature 442, 381 (2006).

[5] E. Yablonovitch, Phys. Rev. Lett. 58, 2059 (1987).

[6] S. John, Phys. Rev. Lett. 58, 2486 (1987).

[7] C. Grillet, P. Pottier, X. Letartre, C. Seassal, P. Rojo-Romeo, P. Viktorovitch, M. Le Vassor d'Yerville, and D. Cassagne, Eur. Phys. J. - Appl. Phys. 16, 37 (2001).
[8] D. Englund, D. Fattal, E. Waks, G. Solomon, B. Zhang, T. Nakaoka, Y. Arakawa, Y. Yamamoto, and J. Vuckovic, Phys. Rev. Lett. 95, 013904 (2005).

[9] M.J. Steel, R. Zoli, C. Grillet, R.C. McPhedran, C. Martijn de Sterke, A. Norton, P. Bassi, and B.J. Eggleton, Phys. Rev. E 71, 056608 (2005).

[10] E. Drouard, H. Hattori, C. Grillet, A. Kazmierczak, X. Letartre, P. Rojo-Romeo, and P. Viktorovitch, Opt. Express 13, 3037 (2005).

[11] Y. Akahane, T. Asano, B.S. Song, and S. Noda, Nature 425, 944 (2003).

[12] K. Srinivasan, P.E. Barclay, M. Borselli, and O. Painter, Phys. Rev. B 70, 081306 (2004).

[13] Z. Zhang and M. Qiu, Opt. Express 12, 3988 (2004).

[14] E. Centeno and D. Felbacq, Phys. Rev. B 62, 7683 (2000).

[15] M. Soljacic, M. Ibanescu, S.G. Johnson, Y. Fink, and J. Joannopoulos, Phys. Rev. E 66, 055601(R) (2002).

[16] T. Tanabe, M. Notomi, S. Mitsugi, A. Shinya, E. Kuramochi, Appl. Phys. Lett. 87, 151112 (2005).

[17] P.E. Barclay, K. Srinivasan, and O. Painter, Opt. Express 13, 801 (2005).

[18] F. Raineri, C. Cojocaru, P. Monnier, A. Levenson, R. Raj, C. Seassal, X. Letartre, and P. Viktorovitch, Appl. Phys. Lett. 85, 1880 (2004).

[19] D. Freeman, S. Madden, and B. Luther-Davies, Opt. Express 13, 3079 (2005).

[20] C. Grillet, D. Freeman, B. Luther-Davies, S. Madden, R. McPhedran, D.J. Moss, M.J. Steel, and B.J Eggleton, Opt. Express 14, 369 (2006).

[21] T.A. Birks and Y.W. Li, IEEE J. Lightwave Technol. 10, 432 (1992).

[22] L. Tong, R.R. Gattass, J.B. Ashcom, S. He, J. Lou, M. Shen, I. Maxwell, and E. Mazur, Nature 426, 816 (2003).

[23] C. Grillet, C. Smith, D. Freeman, S. Madden, B. LutherDavis, E.C. Magi, D.J. Moss, and B.J. Eggleton, Opt. Express 14, 1070 (2006).

[24] I. Hwang, S.K. Kim, J. Yang, S.H. Kim, S. Lee, and Y. Lee, Appl. Phys. Lett. 87, 131107 (2005).

[25] C. Grillet, C. Monat, C. Smith, B.J. Eggleton, D.J. Moss, S. Frédérick, R.L. Williams, D. Dalacu, P.J. Poole, J. Lapointe, and G. Aers, submitted to Opt. Express.

[26] C. Sauvan, P. Lalanne, and J.P. Hugonin, Phys. Rev. B 71, 165118 (2005).

[27] D. Erickson and D. Li, Anal. Chim. Acta 507, 11 (2004).

[28] L. Zhu, Y. Huang, and A. Yariv, Opt. Express 13, 9916 (2005).

[29] P. Mach, M. Dolinski, K.W. Baldwin, J.A. Rogers, C. Kerbage, R.S. Windeler, and B.J. Eggleton, Appl. Phys. Lett. 80, 4294 (2002).

[30] C. Kerbage and B.J. Eggleton, Appl. Phys. Lett. 82, 1338 (2003).

[31] U. Levy, K. Campbell, A. Groisma, S. Mookherjea, and Y. Fainman, Appl. Phys. Lett. 88, 111107 (2006).

[32] D. Erickson, T. Rockwood, T. Emery, A. Scherer, and D. Psaltis, Opt. Lett. 31, 59 (2006).

[33] P. Domachuk, H.C. Nguyen, and B.J. Eggleton, IEEE Photon. Technol. Lett. 16, 1900 (2004).

[34] S.K.Y. Tang, B.T. Nayers, D.V. Vezenov, and G. Whitesides, Appl. Phys. Lett. 88, 061112 (2006).

[35] D.B. Wolfe, D.V. Vezenov, B.T. Mayers, G.M. Whitesides, R.S. Conroy, and M.G. Prentiss, Appl. Phys. Lett. 87, 181105 (2005). 
[36] B.T. Mayers, D.V. Vezenov, V.I. Vullev, and G.M. Whitesides, Anal. Chem. 77, 1310 (2005).

[37] S. Balslev and A. Kristensen, Opt. Express 13, 344 (2005).

[38] S. Balslev, A.M. Jorgensen, B. Bilenberg, K.B. Mogensen, D. Snakenborg, O. Geschke, J.P. Kutter, and A. Kristensen, Lab Chip 6, 213 (2006).

[39] J.C. Galas, J. Torres, M. Belotti, Q. Kou, and Y. Chen, Appl. Phys. Lett. 86, 264101 (2005).

[40] C. Grillet, P. Domachuk, V. Ta'eed, E. Magi, J.A. Bolger, B.J. Eggleton, L.E. Rodd, and J. Cooper-White, Opt. Express 12, 5440 (2004).

[41] P. Domachuk, C. Grillet, V. Ta'eed, E. Magi, J. Bolger, B.J. Eggleton, L.E. Rodd, and J. Cooper-White, Appl. Phys. Lett. 86, 024103 (2005).

[42] C. Monat, P. Domachuk, V. Jaouen, C. Grillet, I. Littler, M. Cronin-Golomb, B.J. Eggleton, S. Mutzenich, T. Mahmud, G. Rosengarten, and A. Mitchell, SPIE conference Optics and Photonics, Optofluidics session, August 2006, San Diego, USA, in: D. Psaltis and Y. Fainman (eds.), Proc. SPIE 6329, 632904 (2006).

[43] N.R. Smith, D.C. Abeysinghe, J.W. Haus, and J. Heikenfeld, Opt. Express 14, 6557 (2006).
[44] B. Maune, M. Loncar, J. Witzens, M. Hochberg, T. BaehrJones, D. Psaltis, A. Scherer, and Y. Qiu, Appl. Phys. Lett. 85, 360 (2004).

[45] H.C. Nguyen, P. Domachuk, B.J. Eggleton, M.J. Steel, M. Straub, M. Gu, and M. Sumetsky, Opt. Express 12, 1528 (2004).

[46] P. Domachuk, H.C. Nguyen, B.J. Eggleton, M. Straub, and M. Gu, Appl. Phys. Lett. 84, 1838 (2004).

[47] P. Mach, T. Krupenkin, S. Yang, and J.A. Rogers, Appl. Phys. Lett. 81, 202 (2002).

[48] A. Ashkin, J.M. Dziedzic, J.E. Bjorkholm, and S. Chu, Opt. Lett. 11, 288 (1986).

[49] D.G. Grier, Nature 424, 810 (2003).

[50] P. Domachuk, M. Cronin-Golomb, B.J. Eggleton, S. Mutzenich, G. Rosengarten, and A. Mitchell, Opt. Express 13, 7265 (2005).

[51] A. Rahmani and P. Chaumet, Opt. Express 14, 6535 (2006).

[52] S.J. Cran-McGreehin, K. Dholakia, and T. Krauss, Opt. Express 14, 7723 (2006).

[53] E. Krioukov, J. Greve, and C. Otto, Sens. Actuators B 90, 58 (2003).

[54] M. Loncar, A. Scherer, and Y. Qiu, Appl. Phys. Lett. 82, 4648 (2003) 